\title{
Miocardiopatía dilatada en el niño
}

\section{Dilated cardiomyopathy in children}

\section{Gonzalo Urcelay ${ }^{\mathrm{a}}$}

aDivisión de Pediatría, Facultad de Medicina, Pontificia Universidad Católica de Chile. Santiago, Chile.

Recibido: 6 de julio de 2020; Aceptado: 26 de julio de 2020

¿Qué se sabe del tema que trata este estudio?

Es una enfermedad miocárdica que afecta la función sistólica ventricular, de etiología múltiple, y que en niños se presenta como causa importante de insuficiencia cardíaca. Existe poca claridad sobre factores pronóstico. El tratamiento está centrado en manejo de la insuficiencia cardíaca.
¿Qué aporta este estudio a lo ya conocido?

Revisión actualizada del tema, incorporando cambios en nomenclatura y clasificación. Aclaración de mecanismos fisiopatológicos con énfasis en etiologías. Se revisan determinantes de pronóstico. Enfoque terapéutico centrado en manejo de la insuficiencia cardíaca basado en evidencia actualizada y recomendaciones de expertos.

\section{Resumen}

Las miocardiopatías (MC) son enfermedades del músculo cardíaco infrecuentes, con una incidencia anual de 1.1-1.2 casos por 100.000 niños. La miocardiopatía dilatada (MCD) es la principal forma, se caracteriza por dilatación ventricular y disfunción sistólica, y es causa importante de insuficiencia cardíaca congestiva (ICC). Las etiologías en niños son múltiples, siendo idiopáticas en el 50\%-70\%. En la evaluación de un niño con MCD es fundamental descartar causas secundarias potencialmente reversibles. El ecocardiograma es la principal herramienta diagnóstica: permite establecer el fenotipo cardíaco, grado de compromiso funcional, y la evolución y respuesta al tratamiento médico. El pronóstico es limitado, siendo mejor en pacientes menores a 1 año al momento de presentación, post miocarditis, o con menor grado de disfunción sistólica ventricular. En los primeros 2 años post presentación alrededor de 20\% tienen normalización de la función ventricular; $40 \%-50 \%$ fallece o requiere un trasplante cardíaco (TC) en los primeros 5 años. El tratamiento médico se basa en recomendaciones de adultos, siendo la evidencia pediátrica muy limitada. El TC es la terapia definitiva en pacientes con ICC terminal, con excelentes resultados a corto y mediano plazo. Una proporción importante de pacientes requiere estabilización en lista de espera, incluyendo asistencia mecánica circulatoria como puente a trasplante. El objetivo de este artículo es actualizar la información disponible en etiología, mecanismos fisiopatológicos, factores pronósticos, y tratamiento de la MCD en niños.
Palabras clave:

Miocardiopatías;

Miocardiopatía

Dilatada;

Trasplante Cardíaco;

Insuficiencia Cardiaca 


\begin{abstract}
Pediatric cardiomyopathies are infrequent diseases of the cardiac muscle, with an annual incidence of 1.1 to 1.2 per 100,000 children. Dilated cardiomyopathy (DCM) is the predominant form, characterized by ventricular dilatation and systolic dysfunction. Etiologies are multiple, with at least $50 \%-70 \%$ of cases being idiopathic. When assessing a child with DCM, secondary potentially reversible causes must be ruled out. The main diagnostic tool is the echocardiogram which allows the identification of cardiac phenotype, to establish the degree of functional compromise, and response to medical therapy. Prognosis is limited but more favorable in infants younger than 1 year at the onset, post myocarditis, or with a lesser degree of ventricular dysfunction. At least $20 \%$ of patients may recover ventricular function in the first 2 years after the onset and $40 \%-50 \%$ may die or need heart transplant in the first 5 years. Medical therapy is mainly based on adult experience with limited scientific evidence in children. Heart transplant is the therapy of choice in patients with end-stage disease, with excellent short- and medium-term survival. A significant proportion of patients may require stabilization on the waiting list, including the use of mechanical circulatory support as a bridge to transplantation. The purpose of this revision is to update the available information on etiology, physiopathological mechanisms, prognostic factors, and management of DCM in children.
\end{abstract}

Keywords:

Heart Diseases;

Dilated

Cardiomyopathy;

Heart Transplantation:

Heart Failure

\section{Introducción}

Las miocardiopatías (MC) son un grupo de enfermedades infrecuentes en Pediatría que afectan al miocardio y se manifiestan por alteración de la función sistólica y/o diastólica, que puede comprometer en forma aislada un ventrículo o ambos. Después de las cardiopatías congénitas, son la principal causa de insuficiencia cardíaca en niños, y se acompañan de importante morbilidad y mortalidad. Luego de la primera clasificación (1980), posteriores reclasificaciones han incorporado nuevas morfologías y avances en etiología $^{1-4}$. En la última (2019), la American Heart Association (AHA) adopta la clasificación basada en primer lugar en las características morfo-funcionales (fenotipo), y luego en las causas o subtipos (genéticas y no-genéticas $)^{5}$ (tabla 1 ). Según diversos estudios poblacionales, el principal fenotipo es la miocardiopatía dilatada (MCD), que ocurre en hasta $50 \%$ del total, seguido por la miocardiopatía hipertrófica ( $\mathrm{MCH})$ en $35 \%$ a $50 \%$, miocardiopatía no compactada (MCNC) en 5\%, y miocardiopatía restrictiva (MCR) en 4\%-5\% ${ }^{5,6}$. Existe además una categoría mixta, en la cual puede haber sobreposición de distintos fenotipos (ej., $\mathrm{MCNC} / \mathrm{MCH}$, $\mathrm{MCNC} / \mathrm{MCD})^{8}$.

El objetivo de este artículo es actualizar la información disponible en etiología, mecanismos fisiopatológicos, factores pronósticos, y tratamiento de la MCD en niños.

\section{Miocardiopatía dilatada}

La MCD es la principal forma de MC, y motivo predominante para requerir un trasplante cardíaco (TC), tanto en niños como en adultos. Se caracteriza por la presencia de dilatación del ventrículo izquierdo (VI) asociado a disfunción sistólica, y grosor normal (o disminuido) de las paredes ventriculares. Es importante descartar una causa que pueda estar asociada a la dilatación y disfunción ventricular, como anatómica, con alteración de la pre o post carga (ej. insuficiencia mitral o coartación aórtica), o isquémica (ej. anomalías coronarias). Si el fenotipo morfo-funcional se mantiene luego de tratada la condición hemodinámica o anatómica, es posible concluir que se trata de una MCD'.

\section{Epidemiología y etiología}

La incidencia anual de las MC es de 1.1-1.2 casos por cada 100.000 niños desde recién nacidos hasta los 18 años $^{5-7}$. La incidencia de MCD es de 0.57 casos por cada 100.000 niños, siendo mayor en hombres que en mujeres $(0,66$ vs 0,47 casos por 100.000), en raza negra (0.98 vs 0.46 casos por 100.000), y en lactantes en comparación con mayores de 1 año $(4,4 v s$ 0,34 casos por 100.000$)^{3,10}$. En Chile no existen estudios poblacionales de MC pediátricas; sin embargo, de acuerdo a la experiencia reportada de TC en niños, el diagnóstico predominante fue la $\mathrm{MCD}^{11}$.

Las causas de MCD pueden ser múltiples y se clasifican en primarias y secundarias (tabla 2). Las MCD primarias son principalmente idiopáticas o familiaresgenéticas. Las MCD secundarias se caracterizan por tener una causa potencialmente tratable y por comprometer múltiples órganos y sistemas, no sólo el corazón. Por otro lado, para que una MCD sea clasificada como primaria es necesario haber descartado las causas secundarias $^{12}$. 
El diagnóstico de MCD idiopática es por exclusión, y se estima que ocurre en el 50\% a 70\% de los casos, proporción que disminuye en la medida que algunas se reclasifican como familiares-genéticas; no existe una característica morfológica o funcional que permita diferenciar una forma idiopática de una familiar ${ }^{10}$. Tampoco existen diferencias en sobrevida en la población pediátrica entre ambas formas de MCD. La evaluación genética es importante en el estudio y clasificación de todas las MC en Pediatría, ya que 42\%-50\% de los casos puede tener un origen genético, entendido como la presencia de algún miembro familiar de primer grado afectado, o un test positivo en un panel genético ${ }^{9,13,14}$.

La MCD familiar ocurre en 30\% a $48 \%$ de los ca$\operatorname{sos}^{15,16}$, con herencia de predominio autosómica dominante, existiendo también la forma autosómica recesiva, ligada a X, y mitocondrial ${ }^{6}$. Los genes causantes codifican 2 subgrupos de proteínas mayores: proteínas del citoesqueleto y del sarcómero ${ }^{17}$. Las proteínas del citoesqueleto identificadas incluyen distrofina, desmina, lamina A/C, $\delta$-sarcoglican, $\beta$-sarcoglican, titina, metavinculina. En las proteínas sarcoméricas los mismos genes responsables de $\mathrm{MCH}$ pueden estar in-

Tabla 1. Clasificación de las miocardiopatías (MC)

Fenotipo
Miocardiopatía dilatada (MCD)
Miocardiopatía hipertrófica (MCH)
Miocardiopatía restrictiva (MCR)
Miocardiopatía arritmogénica (MCA)
No-clasificadas: Miocardiopatía no compactada (MCNC)
Subtipos
Genéticos y no genéticos

Tabla 2. Causas de miocardiopatía dilatada (MCD)

\begin{tabular}{l} 
MCD primaria \\
Familiar/genética \\
Sarcoméricas \\
Enfermedades neuromusculares \\
Enfermedades mitocondriales \\
Laminopatías \\
MCD secundaria \\
Inflamatoria \\
Toxinas \\
Enfermedades metabólicas \\
Enfermedades nutricionales \\
Problemas cardíacos estructurales \\
\hline MCD: miocardiopatía dilatada.
\end{tabular}

volucrados, incluyendo cadena pesada de $\beta$-miosina, proteína $C$ ligada a miosina, actina, $\alpha$-tropomiosina, y troponina cardíaca $\mathrm{T}$ y $\mathrm{C}$. Un nuevo grupo de genes sarcoméricos ha sido identificado, los que codifican proteínas del disco-Z, y de canales iónicos ${ }^{18}$. Las MCD asociadas a mutaciones sarcoméricas corresponden al 10\%-20\% de las MCD heredables, y tienen como resultado una incapacidad en la generación de fuerza del miocito. En general existe poca claridad sobre la interacción entre factores genéticos, ambientales, y otros potenciales modificadores en miocardiopatías pediátricas.

En pacientes con enfermedades neuromusculares las MC son causa de importante morbilidad y mortalidad. Especial importancia tienen las distrofias musculares de Duchenne (DMD) y Becker (DMB); la DMB es una miopatía de menor gravedad que la DMD, con mayores posibilidades de deambular después de la adolescencia. Un 60\%-75\% de pacientes con DMB desarrolla MCD a partir de los 20 años de edad, siendo la miocardiopatía la principal causa de muerte en estos pacientes ${ }^{19}$. Ambas afectan el gen de la distrofina ("distrofinopatías") que se encuentra en el brazo corto del cromosoma X; la herencia es de tipo recesiva ligado a X. El síndrome de Barth es una enfermedad que afecta las mitocondrias a través de un defecto en la cardiolipina. Se presenta típicamente en lactantes de sexo masculino con neutropenia, acidosis, insuficiencia cardíaca, y arritmias. Se produce por mutaciones en el gen tafazina y se asocia a diferentes MC, incluyendo MCD, $\mathrm{MCH}$, y $\mathrm{MCNC}^{20}$. El síndrome de Emery-Dreifuss es causado por una mutación en el gen LMNA (laminopatía) y se presenta como miopatía asociado a $\mathrm{MCD}^{20}$.

Las causas inflamatorias pueden ser infecciosas o no infecciosas, siendo estas últimas muy raras. En niños la miocarditis viral es la principal causa de MCD inflamatoria. Distintos virus pueden ser identificados como causa de miocarditis mediante PCR de genoma viral en sangre, secreciones respiratorias, o tejido miocárdico obtenido por biopsia; actualmente los virus más comúnmente relacionados a miocarditis son adenovirus, enterovirus, parvovirus, herpes virus 6, influenza A y B, varicela zoster, citomegalovirus, y virus Epstein-Barr. El diagnóstico de miocarditis requiere un estudio diagnóstico completo que incluya además de electrocardiograma (ECG) y ecocardiograma, confirmación de inflamación y daño miocárdico, y poder atribuir la inflamación a supuesta infección viral. Esto requeriría la realización de una biopsia miocárdica, método no exento de riesgo en pacientes con insuficiencia cardíaca aguda, con además falsos negativos por inflamación regional. Actualmente como método alternativo se utiliza la resonancia magnética cardíaca, basada en los criterios de Lake Louise para miocarditis $^{21}$. La identificación de partículas de virus median- 
te PCR en sangre o secreciones respiratorias apoya la sospecha diagnóstica cuando el resto de la información clínica es concordante. Un examen complementario de utilidad es la elevación de troponina plasmática.

Otra causa a considerar de MCD es cardiotoxicidad en niños que sobreviven a tratamientos de cáncer, ya sea por uso de antraciclinas y/o radioterapia, especialmente a mayor dosis acumulativa y a menor edad ${ }^{22}$.

\section{Fisiopatología}

En MCD el daño miocárdico se acompaña de disfunción sistólica ventricular, dilatación de las cavidades cardíacas, remodelamiento cardíaco, y frecuentemente insuficiencia mitrálica. A diferencia de adultos, en niños puede existir además compromiso biventricular $^{5}$. Los cambios hemodinámicos van asociados a activación del sistema neurohormonal (sistema nervioso simpático y vía renina-angiotensina-aldosterona) como mecanismo adaptativo. Las alteraciones en las condiciones de carga ventricular son beneficiosas en el corto plazo, pero con el tiempo determinan mayor remodelamiento cardíaco y deterioro en la función ventricular. El conjunto de cambios lleva a la aparición de síntomas y signos propios del síndrome conocido como insuficiencia cardíaca congestiva (ICC) ${ }^{23}$.

\section{Presentación clínica y evaluación diagnóstica}

La presentación clínica de las MC en niños es variable y depende del subtipo específico y severidad del compromiso miocárdico, siendo lo predominante hallazgos de ICC $^{6,7}$. En pacientes con MCD $75 \%$ a $80 \%$ presenta signos y síntomas de ICC, muchos de los cuales pueden confundirse con patología habitual de la niñez como asma, dificultad para alimentarse, vómitos, mal incremento ponderal, y hepatomegalia en lactantes y niños menores ${ }^{6}$. Niños mayores pueden presentar disnea y dolor abdominal, junto con hepatomegalia y evidencias de débito cardíaco comprometido. El espectro de presentación puede variar desde pacientes asintomáticos a insuficiencia cardíaca aguda y shock cardiogénico. Un número importante de pacientes requiere hospitalización al momento del diagnóstico para estabilización y tratamiento de ICC. A diferencia de adultos, la hospitalización de niños por MCD e ICC se asocia a mayor morbilidad y mortalidad, y uso de terapia avanzada de estabilización cardíaca, incluyendo drogas vasoactivas, ventilación mecánica, y ocasionalmente dispositivos de asistencia circulatoria mecánica $^{24}$. Los exámenes habituales como radiografía de tórax y electrocardiograma (ECG) permiten adelantar el posible diagnóstico de MCD. La radiografía típica- mente puede demostrar cardiomegalia y signos de congestión venosa pulmonar, incluyendo edema pulmonar en algunos pacientes. El ECG es inespecífico, pero puede haber taquicardia sinusal y cambios en el voltaje de los complejos QRS; algunos pacientes pueden presentar arritmias como taquicardia supraventricular o ventricular, o alteraciones en la conducción aurículoventricular. El hallazgo de cambios isquémicos incluyendo presencia de ondas $Q$ profundas en derivadas I, aVL, y precordiales V4 a V6 obliga a descartar anomalías coronarias.

En la evaluación de un niño con ICC y sospecha de MC la herramienta diagnóstica principal es la ecocardiografía, ya que permite establecer el fenotipo cardíaco específico, el grado de severidad del compromiso funcional, y la evolución y respuesta a terapia médica. En pacientes con MCD el grado de disfunción y dilatación ventricular puede ser un importante predictor pronóstico, ya sea hacia mayor riesgo de muerte o necesidad de $\mathrm{TC}^{25}$. El fenotipo característico es dilatación de cavidades cardíacas izquierdas asociado a disfunción sistólica (dilatación hipoquinética); es clave por lo tanto en niños ajustar la medición de las cavidades cardíacas a la superficie corporal. Se define dilatación como la presencia de diámetro (o volumen) diastólico de ventrículo izquierdo (DDVI) y diámetro (o volumen) sistólico de ventrículo izquierdo (DSVI) por encima de 2 desviaciones estándar sobre el promedio normal ajustado por superficie corporal ( $>2$ valores $Z$ ). Los parámetros de función sistólica más comúnmente utilizados son la fracción de acortamiento (\%FA, rango normal 28\%-38\%), y fracción de eyección (\%FE, normal > 55\%). En todo primer diagnóstico de MCD, especialmente si se trata de lactantes, es fundamental descartar causas potencialmente tratables, como anomalía de las arterias coronarias, especialmente origen anómalo de la arteria coronaria izquierda desde la arteria pulmonar, y lesiones obstructivas (especialmente coartación aórtica). Cuando la dilatación ventricular izquierda es importante puede acompañarse de dilatación del anillo mitrálico determinando insuficiencia mitrálica y dilatación de la aurícula izquierda.

\section{Pronóstico}

El pronóstico en niños con MCD es pobre. Utilizando como variable clínica mortalidad o TC, alrededor de 40\%-50\% de los pacientes diagnosticados fallece o requiere ser trasplantado a 5 años, y en la mayor parte de los casos los eventos adversos ocurren en los primeros 2 años de presentación ${ }^{10}$. La probabilidad de morir o de requerir un trasplante varía según las características de cada paciente, sin embargo, no existen estudios de estratificación de riesgo. Algunas series 
han identificado 3 factores de buen pronóstico: menor edad, mayor \%FA o \%FE al momento de presentación, y el antecedente de miocarditis ${ }^{25}$. De manera similar se ha establecido como de mayor riesgo el debutar con clínica de ICC, edad mayor a 1 año al momento de presentación, y tener mayor grado de dilatación del VI con peor función sistólica ${ }^{10}$. En pacientes que debutan en ICC el riesgo de morir puede ser tan alto como de $34 \%$ a $1 \mathrm{año}^{26}$. Por etiología, el peor pronóstico y menor probabilidad de trasplantarse lo tienen los pacientes con enfermedades musculares. Existe una proporción de $27 \%-33 \%$ de pacientes con normalización de la función ventricular en un período de 3-15 años posdiagnóstico ${ }^{27}$. El riesgo de muerte súbita (MS) es de 2\%-3\% dentro de los primeros 5 años post diagnóstico, y de hasta $5 \%$ tardíamente ${ }^{28}$.

\section{Manejo médico}

Las estrategias de manejo de la MCD tienen como foco aliviar los síntomas y prevenir las alteraciones hemodinámicas que son causa de la potencial mantención de la ICC como una condición crónica. El número de estudios prospectivos controlados que evalúa la eficacia y seguridad de la terapia de ICC en niños es muy limitado, y gran parte de la información disponible proviene de la extrapolación de la experiencia de terapias en adultos. Las guías disponibles para el manejo de ICC en niños incluyen la clasificación en 4 etapas según condición clínica (A-D) (tabla 3$)^{29}$.

\section{Terapia según etapas de ICC}

ICC en etapa A: Se incluyen en esta etapa niños en riesgo de desarrollar ICC, como puede ocurrir en pacientes sobrevivientes de cáncer expuestos a terapia cardiotóxica (antraciclinas y/o radioterapia), y en niños con DMD. No existe información disponible que apoye el uso profiláctico de inhibidores de la enzima

\section{Tabla 3. Etapas de insuficiencia cardíaca}

\begin{tabular}{cl}
\hline Etapa & Interpretación \\
\hline A & $\begin{array}{l}\text { Pacientes en riesgo de desarrollar ICC, con función cardíaca } \\
\text { normal }\end{array}$ \\
B & Pacientes asintomáticos con función cardíaca anormal \\
C & $\begin{array}{l}\text { Pacientes con función cardíaca anormal, con historia actual o } \\
\text { previa de síntomas de ICC }\end{array}$ \\
D & $\begin{array}{l}\text { Pacientes con ICC avanzada o terminal, con necesidad de infu- } \\
\text { sión continua de inótropos, soporte mecánico, o TC }\end{array}$
\end{tabular}

ICC: insuficiencia cardíaca congestiva; TC: trasplante cardíaco convertidora de angiotensina (IECA) en niños que recibieron terapia cardiotóxica con función cardíaca normal. Algunos estudios randomizados pequeños apoyarían el uso de IECA (como perindropil) en niños con Duchenne ${ }^{30}$.

ICC en etapas B y C: El tratamiento médico de pacientes con ICC crónica incluye una combinación de IECA, $\beta$-bloqueadores, diuréticos, antagonistas de aldosterona, y digoxina. Se utilizan los IECA como vasodilatadores, para disminuir las postcarga elevada por activación del sistema renina-angiotensina-aldosterona. La utilidad de estas drogas está ampliamente establecida en adultos (con nivel de evidencia A), que apoya una mejoría en la sobrevida de pacientes con ICC $^{31}$. En niños, aun cuando los escasos estudios son poco concluyentes ${ }^{32}$, el uso de estas drogas (captopril, enalapril) es considerado como de primera línea y se encuentra incorporado en las guías de manejo de ICC (nivel de evidencia $B)^{29}$. Los $\beta$-bloqueadores antagonizan el efecto deletéreo de la activación simpática crónica sobre el miocardio; carvedilol además por su efecto $\beta$-bloqueador tiene propiedades vasodilatadoras. En adultos, el uso de carvedilol ha demostrado mejorar la sobrevida y disminuir la morbilidad en pacientes con ICC avanzada ${ }^{33}$. En niños los resultados han sido contradictorios, existiendo estudios retrospectivos y prospectivos que apoyarían la mejoría en función ventricular y condición clínica ${ }^{34-36}$. Actualmente se recomienda su incorporación (nivel de evidencia B). En pacientes con signos y síntomas congestivos se utilizan diuréticos, especialmente de asa (furosemida) para alivio sintomático (nivel de evidencia C). El uso de antagonistas de aldosterona está bien establecido en adultos con ICC por disfunción sistólica, tanto por su efecto sobre mortalidad como por sus propiedades antifibróticas y sobre remodelamiento miocárdico ${ }^{37}$. En niños se recomienda incorporar espironolactona en el tratamiento de pacientes con ICC sintomáticos, asociado a IECA y $\beta$-bloqueadores (nivel de evidencia C). Históricamente se ha utilizado digoxina en niños con ICC. Su utilidad se basa en los potenciales efectos sobre inotropismo, atenuación de la respuesta neurohormonal, y control de la frecuencia cardíaca en pacientes adultos. En niños sintomáticos se recomienda su uso a dosis bajas (5-8 $\mu \mathrm{g} / \mathrm{kg}$ al día) con rango ideal de concentración plasmática de $0,5-0,8 \mathrm{ng} / \mathrm{mL}^{38}$ (nivel de evidencia C). Basado en experiencia en adultos, algunos pacientes pediátricos con disfunción sistólica ventricular $(\mathrm{FE}<$ $35 \%$ ), bloqueo completo de rama izquierda, y/o ensanchamiento del intervalo QRS pueden ser candidatos a terapia de resincronización cardíaca ${ }^{39}$ (nivel de evidencia B).

ICC en etapa D: En esta etapa se encuentran pacientes sintomáticos con ICC refractaria a terapia oral optimizada, que requieren con frecuencia manejo 
intrahospitalario, incluyendo drogas vasoactivas (en especial milrinona), y en ocasiones ventilación mecánica. En general la hospitalización se debe a descompensación asociada a comorbilidades (infecciones, anemia, problemas en alimentación, alteraciones hidroelectrolíticas), o compromiso del débito cardíaco y/o sobrecarga de volumen. En algunos casos la descompensación frecuente refleja progresión hacia etapa terminal de la enfermedad, en la cual la sobrevida a largo plazo depende de asistencia circulatoria mecánica, o TC. Los dispositivos de soporte circulatorio se utilizan en pacientes con ICC descompensada en los que la terapia medica sola es insuficiente para mantener la perfusión de órganos y sistemas. Tienen el potencial de mejorar las condiciones generales del paciente en la espera de un TC y en muchos casos de revertir el compromiso secundario (falla renal y/o hepática); sin embargo, su beneficio debe contrastarse con el riesgo de complicaciones tromboembólicas y hemorrágicas ${ }^{40}$. El TC es la terapia de elección en pacientes con ICC terminal refractaria (nivel de evidencia B), y también en algunos pacientes con ICC en etapa $\mathrm{C}$ sintomáticos, con arritmias complejas y alto riesgo de muerte súbita, o con hipertensión pulmonar reversible (nivel de evidencia C) ${ }^{29}$. La sobrevida a 1 año en niños trasplantados es cercana a $92 \%$, a 5 años $80 \%$, y a 10 años $72 \%{ }^{41}$. Esta sobrevida se compara favorablemente con la alta mortalidad reportada en estudios sobre historia natural en niños con MCD, con sobrevida a 1 año de $70 \%$ y a 5 años de $58 \%{ }^{42}$. Sin embargo, la mortalidad en lista de espera es significativa, siendo alta en centros con bajo volumen de trasplantes (cercana al 30\%), y baja (5\%-10\%) en centros de alto volumen ${ }^{43}$. En nuestra experiencia, la mortalidad en lista de espera ha sido de $33 \%$, con un tiempo promedio de espera de 199 días; además 30\% de los pacientes enlistados han requerido asistencia mecánica circulatoria, lo que refleja la complejidad y severidad de los pacientes enlistados ${ }^{8}$. El TC es una opción terapéutica que no está exenta de problemas, incluyendo un pool limitado de donantes, alta mortalidad en la lista de espera, rechazo, y necesidad de inmunosupresión de por vida.

\section{Conclusiones}

Aunque infrecuente, la MCD es la principal forma de MC en Pediatría, y causa importante de ICC. De etiología multifactorial, con predominio idiopático o familiar-genético, se caracteriza por dilatación ventricular y compromiso de la función sistólica. El pronóstico es limitado, con riesgo de fallecer o requerir un TC a 5 años de $40 \%-50 \%$, siendo factores de menor riesgo la edad menor a 1 año, mayor \%FA o \%FE al momento de presentación, y el antecedente de miocarditis. El tratamiento está dirigido a aliviar los síntomas y evitar los cambios hemodinámicos crónicos; el manejo clínico se basa en información proveniente de estudios en adultos. En pacientes con ICC avanzada o terminal el tratamiento definitivo es el TC.

\section{Conflicto de intereses}

El autor declara no tener conflicto de intereses.

\section{Referencias}

1. Report of the WHO/ISFC Task Force on the Definition and Classification of Cardiomyopathies. Br Heart J 1980;44:6723.

2. Report of the 1995 World Health Organization/International Society and Federation of Cardiology Task Force on the Definition and Classification of cardiomyopathies. Circulation 1996;93:841-2.

3. Maron BJ, Towbin JA, Thiene G, et al. American Heart Association, Council on Clinical Cardiology, Heart Failure and Transplantation Committee, Quality of Care and Outcomes Research and Functional Genomics and Translational Biology Interdisciplinary Working Groups, Council on Epidemiology and Prevention. Contemporary definitions and classification of the cardiomyopathies: an American Heart Association
Scientific Statement from the Council on Clinical Cardiology, Heart Failure and Transplantation Committee; Quality of Care and Outcomes Research and Functional Genomics and Translational Biology Interdisciplinary Working Groups; and Council on Epidemiology and

Prevention. Circulation 2006;113:1807-16.

4. Elliott P, Andersson B, Arbustini E, et al. Classification of the cardiomyopathies: a position statement from the European Society of Cardiology Working Group on Myocardial and Pericardial Diseases. Eur Heart J 2008;29:270-6.

5. Lipschultz SE, Law YM, Asante-Korang A, et al. Cardiomyopathy in children: Classification and diagnosis. A Scientific Statement from the American Heart Association. Circulation 2019;140:e9-e68.

6. Lipschultz SE, Sleeper LA, Towbin JA, et al. The incidence of pediatric cardiomyopathy in two regions of the United States. N Engl J Med. 2003;348:1647-55.
7. Nugent AW, Daubeney PE, Chondros $\mathrm{P}$, et al. National Australian Childhood Cardiomyopathy Study. The epidemiology of childhood cardiomyopathy in Australia. N Engl J Med 2003;348:1639-46.

8. Jefferies JL, Wilkinson JD, Sleeper LA, et al. Cardiomyopathy phenotypes and outcomes for children with left ventricular noncompaction: results from the pediatric cardiomyopathy registry. J Card Fail 2015;21:877-84.

9. Lee TM, Hsu DT, Kantor P, et al. Pediatric Cardiomyopathies. Circ Res 2017;121:85573.

10. Towbin JA, Lowe AM, Colan SD, et al. Incidence, causes, and outcomes of dilated cardiomyopathy in children. JAMA 2006;296:1867-76.

11. Becker P, Besa S, Riveros S, et al. Resultados de un programa nacional de trasplante cardíaco pediátrico: fortalezas y debilidades. Rev Chil Pediatr 2017;88:36776. 
12. Arbustini E, Narula N, Dec GW, et al. The MOGE(S) classification for a phenotype-genotype nomenclature of cardiomyopathy: endorsed by the World Heart Federation. J Am Coll Cardiol 2013;62:2046-72.

13. Kindel SJ, Miller EM, Gupta R, et al. Pediatric cardiomyopathy: importance of genetic and metabolic evaluation. J Card Fail 2012;18:396-403.

14. Watkins H, Ashrarafian H, Redwood C. Inherited cardiomyopathies. N Engl J Med 2011;364:1643-56.

15. Towbin JA, Bowles NE. The failing heart. Nature 2002;415:227-33.

16. Rusconi P, Wilkinson JD, Sleeper LA, et al. Differences in presentation and outcomes between children with familial dilated cardiomyopathy and children with idiopathic dilated cardiomyopathy: a report from the Pediatric Cardiomyopathy Registry Study Group. Circ Heart Fail 2017;10:1-12.

17. Kayvanpour E, Sedaghat-Hamedani F, Amr A, et al. Genotype-phenotype associations in dilated cardiomyopathy: meta-analysis on more than 8000 individuals. Clin Res Cardiol 2017;106:127-39.

18. Ware SM. Genetics of pediatric cardiomyopathies. Curr Opin Pediatr 2017;29:534-40.

19. Jefferies JL, Towbin JA. Dilated cardiomyopathy. Lancet 2010;375: 752-62.

20. Feingold B, Mahle WT, Auerbach S, et al. Management of cardiac involvement associated with neuromuscular diseases. A Scientific Statement from the American Heart Association. Circulation 2017;136:e200-e31.

21. Fredrich MG, Sechtem U, Schulz-Menger $\mathrm{J}$, et al. Cardiovascular magnetic resonance in myocarditis: a JACC white paper. J Am Coll Cardiol 2009;53:1475-87.

22. Lipschultz SE, Diamond MB, Franco VI, et al. Managing chemotherapy-related cardiotoxicity in in survivors of childhood cancers. Paediatr Drugs 2014;16:373-89.

23. Ohuchi $H$, Takasugi $H$, Ohashi $H$, et al. Stratification of pediatric heart failure on the basis of neuro-hormonal and cardiac autonomic nervous activities in patients with congenital heart disease. Circulation 2003;108:2368-76.

24. Wittlieb-Weber CA, Lin KY, Zaoutis TE, et al. Pediatric versus adult cardiomyopathy and heart failure-related hospitalizations: a value-based analysis. J Card fail 2015;21:76-82.

25. Alvarez JA, Wilkinson JD, Lipschultz SE. Outcome predictors for pediatric dilated cardiomyopathy: a systematic review. Prog Pediatr Cardiol 2007;23:25-32.

26. Andrews RE, Fenton MJ, Ridout DA, Burch M. New onset heart failure due to heart muscle disease in childhood: a prospective study in the United Kingdom and Ireland. Circulation 2008;117:79-84.

27. Alexander PM, Daubeney PE, Nugent AW, et al. Longterm outcomes of dilated cardiomyopathy diagnosed during childhood: results from a national population-based study of childhood cardiomyopathy. Circulation 2013;128:2039-46.

28. Pahl E, Sleeper LA, Canter CE, et al. Incidence of and risk factors for sudden cardiac death in children with dilated cardiomyopathy: a report from the Pediatric Cardiomyopathy Registry. J Am Coll Cardiol 2012;59:607-15.

29. Kirk R, Dipchand AI, Rosenthal D, et al. International Society for Heart and Lung Transplantation: Guidelines for the management of pediatric heart failure: Executive summary. J Heart Lung Transplant 2014;33:888-909.

30. Duboc D, Meune C, Lerebours G, Devaux J-I, Vaksmann G, Bécane H-M. Effect of perindropil on the onset and progression of left ventricular dysfunction in Duchenne muscular dystrophy. J Am Coll Cardiol 2005;45:855-7.

31. The SOLVD investigators. Effect of enalapril on survival in patients with reduced left ventricular ejection fractions and congestive heart failure. $\mathrm{N}$ Engl J Med 1991;325:293-302.

32. Lewis $\mathrm{AB}$, Chabot $\mathrm{M}$. The effect of treatment with angiotensin-converting enzyme inhibitors on survival of pediatric patients with dilated cardiomyopathy. Pediatr Cardiol 1993;14:9-12.
33. Packer M, Coats AJ, Fowler MB, Katus $\mathrm{H}$, et al. Effect of carvedilol on survival in severe chronic heart failure. $N$ Engl J Med 2001;344:1651-8.

34. Bruns LA, Chrisant MK, Lamour JM, et al. Carvedilol as therapy in pediatric heart failure: an initial multicenter experience. J Pediatr 2001;138:505-11.

35. Azeka E, Franchini JA, Valler C, Alcides BE. Delisting of infants and children from the heart transplantation waiting list after carvedilol treatment. J Am Coll Cardiol 2002;40:2034-2038.

36. Shaddy RE, Boucek MM, Hsu DT, et al. Carvedilol for children and adolescents with heart failure: a randomized controlled trial. JAMA 2007;298:1171-9.

37. The RALES Investigators. Effectiveness of spironolactone added to an angiotensinconverting enzyme inhibitor and a loop diuretic for severe chronic congestive heart failure. The Randomized Aldactone Evaluation Study. Am J Cardiol 1996;78:902-7.

38. Rathore SS, Curtis JP, Wang Y, Bristow MR, Krumholz HM. Association of serum digoxin concentration and outcomes in patients with heart failure. JAMA 2003;289:871-8.

39. Motonga KS, Dubin AM. Cardiac resynchronization therapy for pediatric patients with heart failure and congenital heart disease: A reappraisal of results. Circulation 2014;129:1879-91.

40. Jaqiss RD, Bronicki RA. An overview of mechanical circulatory support in children. Pediatr Crit Care Med 2013;14 (Suppl.1):S3-S6.

41. Pietra BA, Kantor PF, Bartlett HL, et al. Early predictors of survival to and after heart transplantation in children with dilated cardiomyopathy. Circulation 2012;126:107901086.

42. Tsirka AE, Trinkaus K, Chen SC, et al. Improved outcomes of pediatric dilated cardiomyopathy with utilization of heart transplantation. J Am Coll Cardiol 2004;44:391-7.

43. Denfield SW, Azeka E, Das B, et al. Pediatric cardiac waitlist mortality-Still too high. Pediatric Transplantation 2020;24:e13671. 